\title{
Drug Related Hypertension: A Review on an Unappreciated Cause of Blood Pressure Increase
}

QR CODE

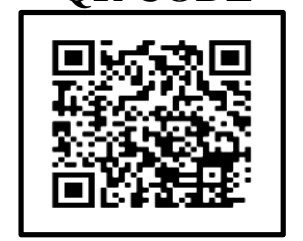

\section{KUNAL MARYA*1, SHIV NAGAR', KUNDAN SHARMA ${ }^{1}$}

Blood pressure elevation is known to be a determining risk factor for cardiovascular disease and mortality. Although blood pressure increase has many causative factors, numerous drugs have also been reported to increase blood pressure. Drugs are often overlooked as a reason of hypertension. A few medications that usually help to reduce blood pressure may increase blood pressure paradoxically or may lead to blood pressure elevation on discontinuation due to a rebound effect. Detailed evaluation of patient's medical therapy may help to identify the culprit drug. Discontinuation of the causative agent is recommended once the drug induced hypertension has been identified. The present review summarizes the therapeutic agents that can induce hypertension and would allow the clinician to recognize this entity and to take the appropriate therapeutic measures.

KEYWORDS: Hypertension, Blood Pressure, Glucocorticoids

\section{INTRODUCTION}

Rapid diagnosis of hypertension can help prevent serious complications such as intracranial bleeding and heart failure. Identification of the intake of these substances may be important because their elimination can obviate the need for unnecessary, costly, and potentially dangerous evaluations, treatments, or both. ${ }^{1}$ Drugs are often overlooked either as a cause of hypertension or as a cause of BP destabilization. Therefore, it is necessary to pay attention to the patient's pharmacological medical history, identify possible drug causes and subsequently evaluate the relevance of the adverse events. Drug related elevation of blood pressure can be caused by various mechanisms including increased intravascular volume due to inadequate hydration or fluid retention, direct or indirect activation of the sympathetic nervous system, direct vascular influence - vasoconstriction. ${ }^{2}$

Drugs that can increase BP include antidepressants, glucocorticoids, thyroid hormones, immunosuppressants, or drugs that lead to mineralocorticoid excess by their mechanism, nonsteroidal anti-inflammatory drugs and sex hormones. Special attention should also be paid to blood pressure elevations during treatment with angiogenesis inhibitors in cancer patients. ${ }^{3}$

\section{ANTI-DEPRESSANT DRUGS}

The effect on blood pressure depends on which mediators and receptors are affected by the antidepressant and the dose of administered drug. Blood pressure elevation is probably caused by their noradrenergic effect. ${ }^{2}$ Tricyclic antidepressants can result in hypertension by affecting noradrenaline and serotonin reuptake, and have other effects such as anticholinergic, antihistamine and alpha-1 lytic, which modulate the overall impact on the cardiovascular system and on the resulting blood pressure. The magnitude of the antidepressant dose administered is important in assessing the association of hypertension with the medication. For amitriptyline, dosulepine, clomipramine and maprotiline hypertension is not reported as an adverse reaction at all, for nortriptyline, the incidence rate of hypertension is reported as an uncommon adverse side effect, and for imipramine, the incidence rate of hypertension is reported as very rare. ${ }^{4}$

The risk of BP elevation and tachycardia when mirtazapine is administered is very low; the probability of occurrence lower than $50 \%$ compared to tricyclic antidepressants is stated. 5 High doses of venlafaxine cause hypertension in more than $12 \%$ of patients. A meta-analysis has shown that BP increase is more 
pronounced in the elderly and in male patients and is dose dependent. ${ }^{5}$ The incidence of elevated diastolic BP $>90 \mathrm{mmHg}$ was statistically and clinically significant only at doses above $300 \mathrm{mg}$ of venlafaxine per day. ${ }^{6}$

\section{ANTINEOPLASTIC AGENTS}

Hypertension is a common side effect that occurs during treatment with agents blocking the vascular endothelial growth factor pathway. These include monoclonal antibodies and tyrosine kinase inhibitors. Binding of VEGF to the respective receptor leads to activation of tyrosine kinase. VEGFR1 and VEGFR2 are mainly localized in endothelial cells. Activation of VEGFR2 triggers regulatory pathways necessary for endothelial biology. The stimulation of phospholipase $\mathrm{C}$ and its associated cascades, which play an important role in cell growth and differentiation, represent the first pathway. The second route leads to activation of phosphatidylinositol-3 kinase - protein kinase B, phosphorylation of endothelial nitric oxide synthase, increased production of nitric oxide and subsequent vasodilation. Third, VEGFR2-mediated activation of cyclooxygenase stimulates the production of vasodilatory prostacyclin., ${ }^{7,8}$ Another route involving cytoplasmic tyrosine kinases influences adhesion and permeability. VEGF also inhibits endothelial production of potent vasoconstrictor endothelinı. VEGF physiological signalling VEGFR2 maintains vascular tone by balancing $\mathrm{NO}$ and prostacyclin induced vasodilation and ET1 regulated vasoconstriction. In connection with the foregoing, agents that block the VEGF pathway may lead to hypertension or BP destabilization. Functional and structural changes in the vascular area are likely mechanisms of hypertension development. Vasoconstriction is enhanced by a decrease in NO and prostacyclin production and an increase in endothelinı production. ${ }^{9}$

Renal dysfunction is not the initial cause of hypertension in patients treated with VEGF inhibitors, but inhibition of renal NOS associated with impaired sodium excretion followed by fluid retention may contribute to the development of hypertension..$^{7,8,10}$ Blood pressure elevation is rapid in most patients; blood pressure should be monitored closely especially during the first 3-4 weeks after initial drug administration and resolves after withdrawal. In patients with advanced cancer, elevated BP was detected with sorafenib therapy on the first day of treatment and fully expressed at the time when balanced drug concentrations were reached around day
7. ${ }^{11}$ Not only do patients face the risk of developing hypertension when starting treatment with VEGF pathway inhibitors but they are also at risk of developing hypotension upon withdrawal. Patients receiving therapy with VEGF-blocking drugs are often polymorbid and have an extensive cardiological history and medication. In all patients on a VEGF-blocking medication, newly introduced medication should be considered as a possible cause of hypertension in the differential diagnosis of sudden BP destabilization, the severity of the adverse side effect should be evaluated, and the relevant course of action should be followed accordingly. ${ }^{12}$

\section{GLUCOCORTICOIDS}

The incidence of hypertension in patients with Cushing's syndrome is $70-80 \%$, but only $15-20 \%$ in patients treated with high doses of synthetic corticosteroids that have lower mineralocorticoid activity than cortisol. ${ }^{13}$ Oxidative stress and nitric oxide deficiency may be involved in the pathogenesis of glucocorticoid-induced hypertension. ${ }^{14}$ However, the exact mechanism of glucocorticoid-induced hypertension is still not fully elucidated and appears to be multifactorial.

\section{IMMUNOSUPPRESSANTS}

The incidence of hypertension in cyclosporine therapy is $50 \%$ and $35 \%$ for tacrolimus. ${ }^{5}$ Everolimus and temsirolimus have antiproliferative effects which are used not only in transplantology to reduce lymphocyte proliferation, but also in oncology to influence tumor cell proliferation. The incidence of hypertension in both drugs has been reported in studies from 1 to $10 \% .^{15}$ The mechanism of pressure elevation includes changes in the level of vascular endothelial function, decreased levels of vasodilatory mediators (prostacyclin and nitric oxide), increased levels of vasoconstrictive endothelin and increased insulin resistance.

\section{ERYTHROPOIETIN}

Hypertension may develop in $20-30 \%$ of patients receiving erythropoietin, occurring 2 weeks to 4 months after initiation of treatment. The anticipated mechanism of this side effect is not exactly known, with increased calcium in vascular smooth muscle cells, activation of the local renin angiotensin aldosterone system, increased ET1 production and reduced NO synthesis playing a certain role (2). Erythropoietin may increase BP by more than $10 \mathrm{mmHg}$, more often in patients on dialysis than in patients not yet on dialysis. ${ }^{2}$ 


\section{DRUGS AFFECTING THE EFFECT OF MINERALOCORTICOIDS}

Drugs interfering with corticoid metabolism by influencing the activity of some important enzymes may contribute to hypertension. The antifungal agent posaconazole may lead to an excess of mineralocorticoids in the body by its mechanism of action. ${ }^{16}$ Also, abiraterone, which is administered to patients with castration-resistant prostate cancer, leads to clinically significant corticoid imbalance in the body, cortisol deficiency, and an excess of mineralocorticoids. ${ }^{17}$

\section{NSAIDS}

A meta-analysis conducted in the 1990s showed that non-steroidal anti-inflammatory drugs (NSAIDs) used more than 1 week in uncomplicated hypertensive and normotensive patients increased BP by an average of 5 mmHg. ${ }^{2}$ The exact mechanism by which NSAIDs contribute to BP elevation is not fully understood and is multifactorial. NSAIDs inhibit cyclooxygenase 1 and 2, thus reducing prostaglandin synthesis, reducing $\mathrm{NO}$ and increasing ET1. NSAIDs reduce the efficacy of some antihypertensive drugs such as diuretics, betablockers and ACE inhibitors, but do not interfere with the action of calcium antagonists and centrally acting antihypertensive drugs. ${ }^{18}$

\section{SEX HORMONES}

Hypertension is 2-3 times more common in women using oral contraceptives than in the control group. ${ }^{19}$ Estrogens and progestins are believed to increase the synthesis of angiotensinogen in the liver, thereby increasing the production of angiotensin II and the secretion of aldosterone, which activates the mineralocorticoid receptor and causes sodium resorption and water retention. Testosterone causes increased sodium and water retention through androgen receptor agonism. ${ }^{2}$

\section{CONCLUSION}

With exponential growth in pharmaceutical industry, several drugs which are generally perceived as a potential cause of hypertension development are now being used for prophylactic and therapeutic use across the globe. New salts that interfere with the pharmacodynamic effect of the body's regulatory mechanisms are already in the market and attention should be paid to this issue. Most patients with high blood pressure have essential hypertension or wellknown forms of secondary hypertension such as renal parenchymal disease, renal artery stenosis, hyperaldosteronism, or pheochromocytoma. Healthcare professionals are not too aware of drug associated increase in blood pressure. An accurate and detailed medical history detailing all medications being taken by the patient concomitantly or in past would help to identify the culprit drug in case of drug related hypertension. Identification of the intake of these substances may be important because their elimination can prevent the need for unnecessary, costly, and potentially dangerous evaluations, treatments, or both.

\section{REFERENCES}

1. Elliott WJ. Drug interactions and drugs that affect blood pressure. J Clin Hypertens (Greenwich). 2006;8(10):731-7.

2. Lovell AR, Ernst ME. Drug-Induced Hypertension: Focus on Mechanisms and Management. Curr Hypertens Rep. 2017; 19:39.

3. Salvetti M, Paini A, Bertacchini F, Stassaldi D, Aggiusti C, Agabiti Rosei C, et al. Acute blood pressure elevation: Therapeutic approach. Pharmacological Research.2018; 130:180-90.

4. Scalco AZ, Scalco MZ, Azul JB, Lotufo Neto F. Hypertension and depression. Clinics 2005;60(3):24150.

5. Sander GE. Secondary hypertension Drugs and herbal preparations that increase pressure. Journal of the American Society of Hypertension. 2014; 8(12):946-8.

6 . Thase ME. Effects of venlafaxine on blood pressure: a meta-analysis of original data from 3744 depressed patients. J Clin Psychiatry 1998; 59(10): 502-508.

7. Touyz RM, Lang NN, Herrmann J, van den Meiracker AH, Danser AHJ. Recent Advances in Hypertension and Cardiovascular toxicities with VEGF Inhibition. Hypertension. 2017; 70:220-6.

8. Touyz RM, Herrmann SMS, Herrmann J. Vascular toxicities with VEGF inhibitor therapies-focus on hypertension and arterial thrombotic events. Journal of the American Society of Hypertension. 2018; 12(6): 40925.

9. Agarwal M, Thareja N, Benjamin M, Akhondi A, Mitchell GD. Tyrosine Kinase Inhibitor-Induced Hypertension. Curr Oncol Rep. 2018; 20(8):65.

10. Hayman SR, Leung $\mathrm{N}$, et al. VEGF Inhibition, Hypertension, and Renal Toxicity Curr Oncol Rep. 2012 August; 14(4): 285-294.

11. Maitland, ML, Kasza KL, et al. Ambulatory monitoring detects sorafenib-induced blood pressure elevations on the first day of treatment. Clin Cancer Res. 2009; 15(19): 6250-6257.

12. Colevas AD, Setser A. The NCI Common Terminology Criteria for Adverse Events (CTCAE) v 3.0 
is the new standard for oncology clinical trials. Journal of Clinical Oncology. 2004;22(14_suppl): 6098-6098. 13. Isidori AM, Graziadio C, Paragliola RM, Cozzolino A, Ambrogio AG, Colao A, et al. The hypertension of Cushing's syndrome: controversies in the pathophysiology and focus on cardiovascular complications. Journal of Hypertension. 2015; 33: 44-6o. 14. Hattori T, Murase T, Erika Iwase, Keiji Takahashi, Masafumi Ohtake, Koji Tsuboi, et al. Glucocorticoid-induced hypertension and cardiac injury: effects of mineralocorticoid and glucocorticoid receptor antagonism. Nagoya J. Med. Sci.2013; 75: 81-92. 15. Eisen T, Sternberg CN, Robert C, Mulders P, Pyle L, Zbinden S, et al. Targeted Therapies for Renal Cell Carcinoma: Review of Adverse Event Management Strategies. J Natl Cancer Inst 2012; 104: 93-113.
16. Boughton Ch, Taylor D, et al. Mineralocorticoid hypertension and hypokalaemia induced by posaconazole. Endocrinol Diabetes Metab Case Rep. 2018; 17-0157.

17. Auchusa RJ, Yub MK, et al. Use of Prednisone With Abiraterone Acetate in Metastatic castration-Resistant Prostate Cancer. The Oncologist 2014; 19: 1231-1240.

18. Grossman A, Messerli FH, et al. Drug induced hypertension - An unappreciated cause of secondary hypertension. European Journal of Pharmacology. 2015; 763: 15-22.

19. Gyamlani G, Gerac SA. Secondary Hypertension due to Drugs and Toxins. Southern Medical Journal. 2007; 7(100): 692-9.

Source of support: Nil, Conflict of interest: None declared

Cite this article as:

Marya K, Nagar S, Sharma K. Drug Related Hypertension: A Review on an

Unappreciated Cause of Blood Pressure Increase. Int Healthc Res J. 2020;3(12):376-379. https://doi.org/10.26440/IHRJ/0312.03196 